\title{
Use of Bony Landmarks during Adrenal Venous Sampling to Guide Catheterization of the Left Adrenal Vein
}

\author{
Lucas R. Cusumano ${ }^{1}$ Sipan Mathevosian ${ }^{1} \quad$ Joshua K. Sweigert ${ }^{1} \quad$ Ravi N. Srinivasa $^{1} \quad$ Aarti P. Luhar ${ }^{1}$ \\ John M. Moriarty ${ }^{1}$ \\ ${ }^{1}$ Division of Interventional Radiology, Department of Radiology, \\ David Geffen School of Medicine at UCLA, Los Angeles, California, \\ United States \\ Address for correspondence John M. Moriarty, MD, Ronald \\ Reagan UCLA Medical Center, 10945 LeConte Avenue, Los Angeles, \\ CA 90095, United States (e-mail: jmoriarty@mednet.ucla.edu).
}

\begin{abstract}
Keywords

- adrenal venous sampling

- hyperaldosteronism

- adrenal adenomas

Purpose The aim of this study was to examine the utility of fluoroscopic bony landmarks in predicting the location of the left adrenal vein during adrenal vein sampling (AVS).

Methods Eighty-six AVS procedures were performed in 81 patients between August 2013 and March 2020. A selectivity index was calculated for each case by dividing the measured left adrenal vein cortisol level by the peripheral vein cortisol level. Successful "target" left adrenal vein catheterization was confirmed in cases with a selectivity index of three or greater. Intraprocedural AVS fluoroscopic images were selected that demonstrated catheter position in the left adrenal vein. Lateral distance from the catheter tip in the left adrenal vein to the lateral margin of the left pedicle at the associated vertebral body level was measured.

Results Mean patient age was 56.4 years (range: 19-80 years) and 48 (59.3\%) patients were male. Target sampling in the left adrenal vein was confirmed in 82 (95.3\%) cases. In 78 (95.1\%) targeted cases, the catheter terminated less than $25 \mathrm{~mm}$ from the left lateral pedicle at a mean distance of $11.2 \mathrm{~mm}$. The catheter was most frequently placed at the T12 and L1 vertebral body levels. Four (4.7\%) cases demonstrated nontarget catheter positioning, two (50.0\%) of these cases were within $25 \mathrm{~mm}$. Conclusion The position of the left adrenal vein is generally located in a predictable position relative to bony landmarks. By utilizing these landmarks, positioning of the sampling catheter during AVS can be more reliable with the potential to avoid repeat procedures and delays in patient care.
\end{abstract}

\section{Introduction}

Adrenal vein sampling (AVS) is the gold standard technique for establishing laterality of aldosterone secreting adrenal adenomas and guiding potentially curative adrenalectomy in patients with primary aldosteronism and secondary hypertension..$^{1-3}$ Adrenalectomy performed in conjunction with AVS is more successful in curing hypertension, reducing the need for antihypertensive medications and potassium supplementation. ${ }^{4}$ published online June 4, 2021
DOI https://doi.org/

$10.1055 / \mathrm{s}-0041-1730113$ ISSN 2542-7075
(C) 2021. The Pan Arab Interventional Radiology Society

This is an open access article published by Thieme under the terms of the Creative Commons Attribution-NonDerivative-NonCommercial-License, permitting copying and reproduction so long as the original work is given appropriate credit. Contents may not be used for commercial purposes, or adapted, remixed, transformed or built upon. (https://creativecommons.org/licenses/by-nc-nd/4.0/). Thieme Medical and Scientific Publishers Private Ltd. A-12, Second Floor, Sector -2, NOIDA -201301, India 
AVS has a high technical success rate ranging from 80 to over $95 \%$ in the hands of experienced operators. ${ }^{4-7}$ The procedure may, however, be challenging with high radiation doses and failures mostly attributed to technical factors. ${ }^{8,9}$ Several prior studies have investigated facilitating cannulation using preoperative computed tomography (CT) mapping, intraoperative $\mathrm{CT}$ angiography, and different wire-catheter selection techniques. ${ }^{6,9-13}$ While right-sided cannulation is considered more cumbersome with higher failure rates, left-sided failures occur in as many as $39 \%$ of unsuccessful cases. ${ }^{6,14}$

Reliable landmarks allow improved confidence of correct catheter placement and hence improved "target" sampling of the desired vessel. The inferior accessory hepatic vein and inferior emissary veins have previously been described as anatomic markers for the right adrenal vein. ${ }^{15,16}$ Fluoroscopic bony landmarks have also shown value in facilitating other venous interventions. ${ }^{17}$ No such landmarks have been described for the left adrenal vein. The focus of this investigation is the utility of fluoroscopic bony landmarks in predicting the location of the left adrenal vein during AVS.

\section{Materials and Methods}

\section{Study Cohort}

This retrospective study was approved by the institutional review board and informed consent was waived. Patients who had an AVS procedure performed at our institution between August 2013 and March 2020 were identified using the Montage Search and Analytics software (Montage Healthcare Solutions, Philadelphia, Pennsylvania, United States). Demographic and laboratory data were collected from chart review of each patient in this population, including age, sex, and body mass index, as well as cortisol and aldosterone levels for the inferior vena cava and left adrenal vein taken during the procedure. A selectivity index was calculated for each case by dividing the measured left adrenal vein cortisol level by the peripheral vein cortisol level. ${ }^{18}$ Cases with a selectivity index of three or greater were determined to have had successful "target" left adrenal vein catheterization. Procedures with a selectivity index less than three were considered unsuccessful with "non-target" vessel sampling. ${ }^{3}$

\section{Procedure}

All patients signed an informed consent form prior to the procedure, which described the AVS procedure and all predictable complications of the procedure were discussed in-person with the patient, including groin hematoma, adrenal hemorrhage, thrombosis, infection, contrast reaction, and possibility of nondiagnostic test results. AVS was performed by a single experienced interventional radiologist, with 12 years of AVS performance.

All procedures were performed under conscious sedation and local anesthesia. Per institutional protocol, poststimulation sampling only was performed. Immediately prior to the procedure, a stimulant bolus injection of $250 \mu \mathrm{g}$ cosyntropin was administered through a peripheral intravenous line, followed by a 20 -minute infusion of $250 \mu \mathrm{g}$ cosyntropin in $0.9 \%$ normal saline. Under ultrasound guidance and using a 5 -French micropuncture access kit, the common femoral veins bilaterally were accessed. On the right side, a 6-French sheath was advanced over a 0.035 " guidewire into the right common iliac vein, and on the left side, a 7-French renal double curve (RDC) catheter (Boston Scientific, Marlborough, Massachusetts, United States) was advanced into the inferior vena cava. The RDC catheter was manipulated into the left renal vein and left renal venography performed. Using a 4-French angled glide catheter and 0.035" Glidewire (Terumo, Somerset, New Jersey, United States) attempts were made to subselect the left adrenal vein from the common trunk with the left inferior phrenic vein. Subsequent sampling was performed for aldosterone and cortisol. The catheter was secured in position. On the right side, a 5-French RDC catheter (Boston Scientific) was used to select the right renal vein and right hepatic veins and the position was marked with venography. Subsequently, the right adrenal vein was selected and gentle venography performed. Sampling of the peripheral circulation, right and left adrenal veins was then performed simultaneously for both serum aldosterone and cortisol. Rapid cortisol assays were not utilized during the procedure. The catheters, guidewire, and sheath were then removed. Hemostasis was achieved with manual compression. Fluoroscopy time and air kerma were recorded for each procedure.

\section{Sampling Catheter Position}

For identification and measurements of the left AVS, catheter position fluoroscopic images were evaluated using a GE Centricity PACS system (GE Healthcare, Barrington, Illinois, United States). Intraprocedural AVS fluoroscopic images were selected that demonstrated catheter position in the left adrenal vein at the time of sampling. In all cases, a strict anteroposterior fluoroscopy image centered over the lumbar vertebrae was used for evaluation. Measurements were taken at the end of expiration to control for respiratory motion. Lateral distance from the catheter tip in the left adrenal vein to the lateral margin of the left pedicle at the associated vertebral body level was measured ( $\boldsymbol{- \text { Fig. }} \mathbf{1}$ ). The associated vertebral body level of the catheter position was also recorded, as well as the relative position within that vertebral body (upper, middle, lower).

\section{Statistical Analysis}

Continuous data are expressed as mean \pm standard deviation. Comparisons between groups were made using the Fisher's exact test for categorical variables and Mann-Whitney analysis for continuous variables. $p$-Values $<0.05$ were considered significant. Statistical analyses were performed using a computer using GraphPad Prism, version 8.4 (GraphPad Software, La Jolla, California, United States).

\section{Results}

\section{Patient Characteristics}

Eighty-six AVS procedures were performed in 81 patients between August 2013 and March 2020. Patients were between 19 and 80 years at the time of the procedure with a mean age of 56.4 ( \pm 11.3 ). Forty-eight (59.3\%) patients were male and 


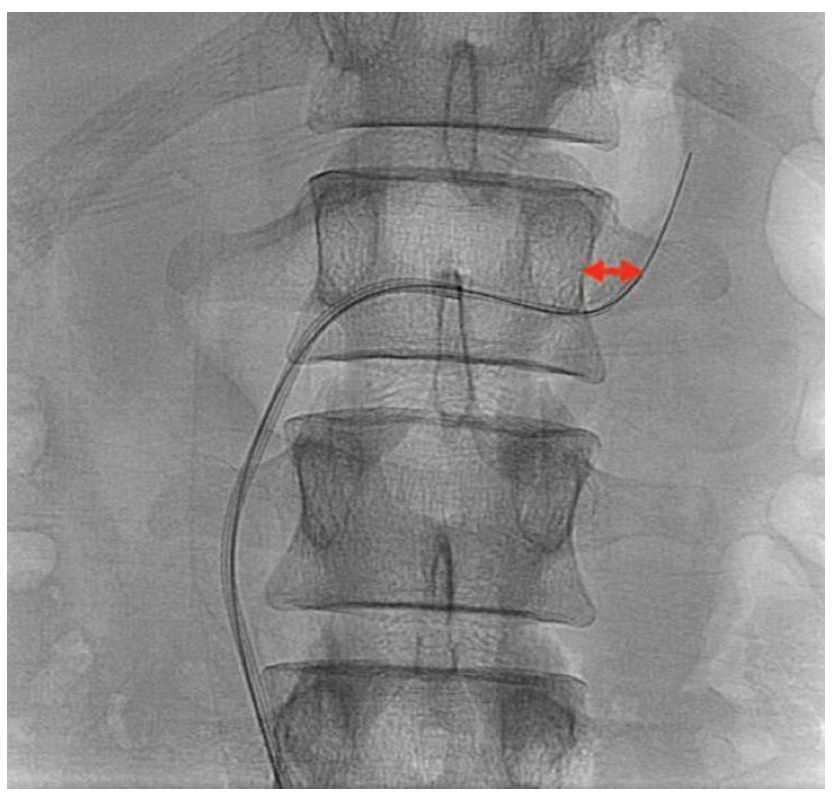

Fig. 1 Representative image of sampling catheter position measurement. Red arrow represents distance between lateral border of the left pedicle and sampling catheter tip.

$33(40.7 \%)$ were female. Mean body mass index was 29.8 ( \pm $6.9) \mathrm{kg} / \mathrm{m}^{2}$. Mean fluoroscopy time was $13.1( \pm 10.8)$ minutes with a mean air kerma of $892.9( \pm 1113.8)$ mGy. Target sampling in the left adrenal vein was confirmed with a selectivity index greater than three in 82 (95.3\%) cases. Four (4.7\%) cases demonstrated a selectivity index of less than three indicating nontarget catheter positioning.

\section{Sample Catheter Position}

In the 82 cases with successful left AVS, the catheter was most frequently placed at the T12 and L1 vertebral body levels (-Fig. 2). Mean distance from the left lateral pedicle was $10.5( \pm 7.6) \mathrm{mm}$ and $10.6( \pm 7.4)$ at the $\mathrm{T} 12$ and L1 vertebral body level, respectively ( - Table 1 ). In the one patient with the catheter positioned at the T11 vertebra body level, the catheter was located $12.7 \mathrm{~mm}$ away from the left lateral pedicle. At the $\mathrm{L} 2$ vertebral level, the mean distance was $15.0( \pm 16.6) \mathrm{mm}$.

In all of the four nontargeted cases, the AVS catheter was placed at the T12 and L1 vertebral body levels ( - Fig. 3 ). Overall mean distance from the left lateral pedicle was 22.6 $( \pm 20.0) \mathrm{mm}$. In two cases, the catheter placement was greater than $25 \mathrm{~mm}$ from the lateral pedicle. Due to the distance

Table 1 Mean catheter tip distance from the left lateral pedicle in targeted left AVS cases

\begin{tabular}{|l|l|}
\hline Vertebral body level & $\begin{array}{l}\text { Mean distance from lateral } \\
\text { pedicle }(\mathbf{m m})\end{array}$ \\
\hline T11 & 12.7 \\
\hline T12 & $10.5( \pm 7.6)$ \\
\hline L1 & $10.6( \pm 7.4)$ \\
\hline L2 & $15.0( \pm 16.6)$ \\
\hline
\end{tabular}

Abbreviation: AVS, adrenal vein sampling.

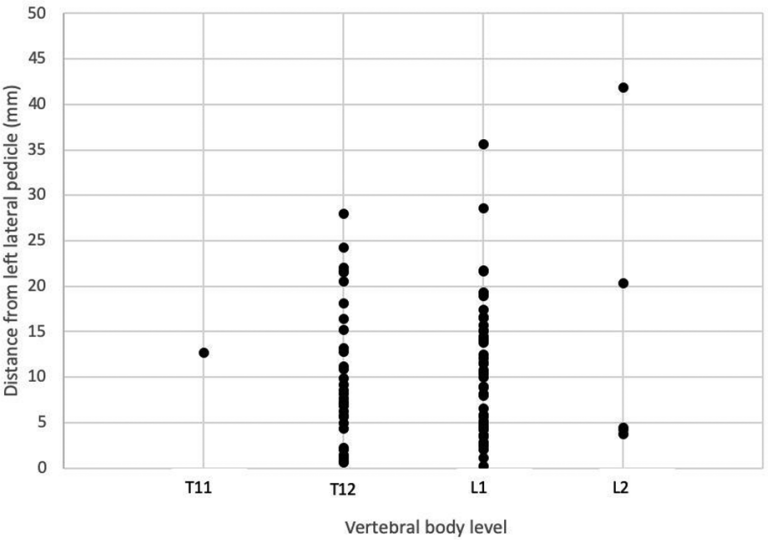

Fig. 2 Sampling catheter position in targeted left adrenal vein sampling cases.

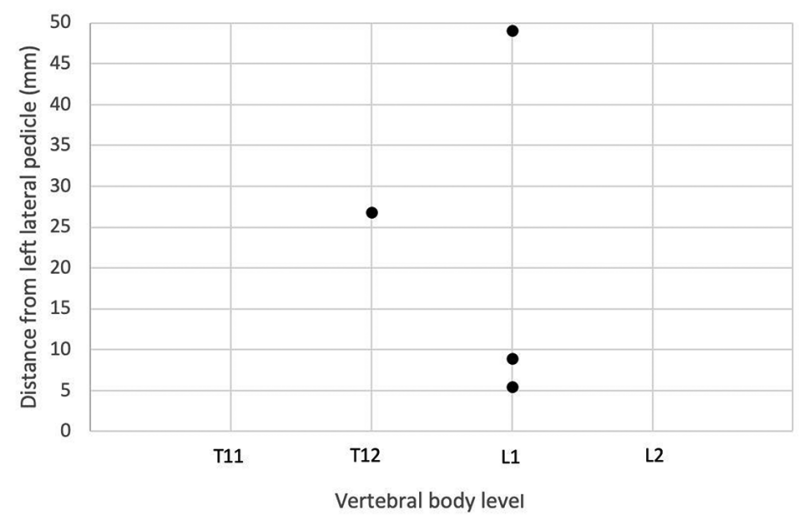

Fig. 3 Sampling catheter position in nontargeted left adrenal vein sampling cases.

away from the lateral pedicle, it is suspected that the catheter tip was located either in a left inferior phrenic vein or a branch of the left renal vein. In two cases, the catheter placement was less than $10 \mathrm{~mm}$ from the left lateral pedicle. The precise vessel location of the sampling catheter in these cases is indeterminant on retrospective review; however, the selectivity index less than three makes positioning within the left adrenal vein unlikely.

\section{Discussion}

AVS is an important step prior to consideration of adrenalectomy in patients with hyperaldosteronism because when unilateral adrenal disease is identified it can provide patients with the option of a unilateral adrenalectomy. ${ }^{1}$ Successful target sampling of the right and left adrenal veins is necessary to unequivocally determine laterality. ${ }^{3}$ Interventionalists at high volume centers may see success rates up to $95 \%{ }^{4-7}$ Although we only present data regarding left adrenal vein AVS, successfully targeting the left adrenal vein in $95.3 \%$ of cases is compatible with the rates seen in other high volume centers. Most unsuccessful AVS procedures are due to operator failures. ${ }^{6}$ However, increased experience, instituting standard operating procedures, and/or intraprocedural confirmatory tests can result in greater success rates. ${ }^{14}$ 
Anatomy of the left adrenal vein is fairly consistent. From its origin in the adrenal hilum, it typically travels inferomedially to form a common trunk with the inferior phrenic vein and then drain into the left renal vein., ${ }^{719}$ Variations occur in less than $10 \%$ of patients and most commonly includes duplication of the left adrenal vein or drainage of the left adrenal vein directly into the left renal vein. ${ }^{20}$ We found that intraprocedural left renal venogram was sufficient to identify the anatomy of the left adrenal vein and no variant anatomy was observed in our cohort. As previously described, the common trunk of the left adrenal vein and inferior phrenic vein is most frequently selected from the left renal $\sim 3$ to $4 \mathrm{~cm}$ distal to the inferior vena cava by positioning the catheter tip cephalad. ${ }^{7}$ In all cases, we achieved bilateral or dual unilateral access to perform simultaneous sampling of both the right and left adrenal veins. This allowed us to account for pulsatile variations in adrenal gland secretion and prevent time-related variability that could occur in sequential sampling (i.e., sampling the adrenal glands one at time). ${ }^{3}$ Nevertheless, most institutions perform sequential sampling that may avoid potential complications such as adrenal vein thrombosis because the sampling catheter remains in the adrenal vein for a shorter period of time. ${ }^{3,21}$ However, in our cohort we did not observe any complications.

In all targeted left AVS cases, the catheter tip was lateral to the left pedicle. Left AVS was most commonly performed at the T12 and L1 vertebral with a mean distance of approximately $11 \mathrm{~mm}$ from the left lateral pedicle. Targeted left AVS further than $25 \mathrm{~mm}$ is less likely as we observed $95.1 \%$ of targeted cases within this distance. In the two nontargeted cases with the catheter tip greater than $25 \mathrm{~mm}$, we retrospectively suspect that the catheter tip was located either in a left phrenic vein or a branch of the left renal vein. Nontargeted left AVS can also occur within $10 \mathrm{~mm}$, as we observed in two cases. The precise vessel location of the sampling catheter in our nontargeted AVS cases within $10 \mathrm{~mm}$ is indeterminant; however, we suspect that the catheter may have migrated back into the left renal vein prior to sampling.

A study evaluating failures in AVS found that $31 \%$ of failures were left sided. ${ }^{6}$ The authors determined that all purely left-sided failures were due to hemodilution and by lowering their selectivity index cutoff value from five to three, they found all left sided failures to be successful. ${ }^{6}$ Cutoff values for selectivity index vary among institutions, although most utilize a selectivity index threshold between three and five if cosyntropin stimulation was performed. ${ }^{21}$ We chose a cutoff value of three or greater for consistency with consensus guidelines. ${ }^{3}$ In our four nontargeted left AVS cases, the selectivity index was less than two and it is unlikely that hemodilution was the reason for failure.

Use of other adjuncts to AVS may improve procedural efficacy. For example, preprocedural imaging with CT venography can allow for prior evaluation of the vascular anatomy to guide sampling catheter position. ${ }^{22}$ Whenever possible available preprocedural imaging was reviewed to evaluate for uncommon variants. However, we did not routinely perform dedicated preprocedure CT venography because it would likely have negligible benefit in our cases since we were able to readily identify and successfully select the bilateral adrenal veins in nearly all cases. C-arm CT may offer intraprocedural evaluation of the vascular anatomy without the need of prior dedicated imaging and provide guidance in locating and reposition the sampling catheter. ${ }^{23}$ Intraprocedural cortisol levels may similarly offer the opportunity of immediate intraprocedural feedback and the opportunity to adjust sampling catheter position. ${ }^{3,24}$ We did not utilize intraprocedural cortisol levels or C-arm CT primarily due to concerns of added procedure time and cost. Nevertheless, in cases of variant anatomy or sampling catheter position further than expected from bony or vascular landmarks, the benefits of these techniques may outweigh concerns.

Our study had limitations inherent to retrospective studies performed at a single academic center by a single interventional radiologist. Anatomical variations in the drainage of the left adrenal vein may limit the applicability of our findings as no variant anatomy was visualized by fluoroscopy. All measurements were made in a strict anteroposterior projection; however, oblique rotation of the fluoroscopy machine may alter the perceived distance of the sampling catheter from the pedicle. In addition, variations in thoracolumbar spinal anatomy such as severe scoliosis or absence of the pedicle may lead to infeasibility of using this technique.

In conclusion, the position of the left adrenal vein is generally located in a predictable position relative to bony landmarks. By utilizing these landmarks, positioning of the sampling catheter can be more reliable with the potential to avoid repeat procedures and delays in patient care. In cases where catheter position is outside of the expected location relative to these bony landmarks, additional confirmative techniques can be performed to minimize procedure time and cost if these techniques were otherwise applied ubiquitously.

\section{Source of Support}

None.

\section{Conflicts of Interest}

Outside the submitted work Dr. Moriarty reports personal fees from Argon Medical, grants and personal fees from Angiodynamics Inc., personal fees from Boston Scientific Inc., grants and personal fees from Penumbra Inc., personal fees from BD Bard, personal fees from Thrombolex Inc., personal fees from PAVmed Inc. The other authors have no additional relevant disclosures.

\section{References}

1 Funder JW, Carey RM, Mantero F, et al. The management of primary aldosteronism: case detection, diagnosis, and treatment: an endocrine society clinical practice guideline. J Clin Endocrinol Metab 2016;101(5):1889-1916

2 Young WF, Stanson AW, Thompson GB. Grant CS, Farley DR, van Heerden JA. Role for adrenal venous sampling in primary aldosteronism. Surgery 2004;136(6):1227-1235

3 Rossi GP, Auchus RJ, Brown M, et al. An expert consensus statement on use of adrenal vein sampling for the subtyping of primary aldosteronism. Hypertension 2014;63(1):151-160

4 Rossi GP, Rossitto G, Amar L, et al. Clinical outcomes of 1625 patients with primary aldosteronism subtyped with adrenal vein sampling. Hypertension 2019;74(4):800-808 
5 Jakobsson H, Farmaki K, Sakinis A, Ehn O, Johannsson G, Ragnarsson O. Adrenal venous sampling: the learning curve of a single interventionalist with 282 consecutive procedures. Diagn Interv Radiol 2018;24(2):89-93

6 Trerotola SO, Asmar M, Yan Y, Fraker DL, Cohen DL. Failure mode analysis in adrenal vein sampling: a single-center experience. J Vasc Interv Radiol 2014;25(10):1611-1619

7 Daunt N. Adrenal vein sampling: how to make it quick, easy, and successful. Radiographics 2005;25(Suppl 1) :S143-S158

8 Fuss CT, Treitl M, Rayes N, et al. Radiation exposure of adrenal vein sampling: a German multicenter study. Eur J Endocrinol 2018;179(4):261-267

9 Harsha A, Trerotola SO. Technical aspects of adrenal vein sampling. J Vasc Interv Radiol 2015;26(2):239

10 Degenhart C, Strube H, Betz MJ, et al. CT mapping of the vertebral level of right adrenal vein. Diagn Interv Radiol 2015;21(1):60-66

11 Oguro S, Nakatsuka S, Yashiro H, et al. CT during arteriography to visualize the right adrenal vein for adrenal venous sampling. J Vasc Interv Radiol 2015;26(6):910-914

12 Andrews JC, Thompson SM, Young WF. A coaxial guide wire-catheter technique to facilitate right adrenal vein sampling: evaluation in 76 patients. J Vasc Interv Radiol 2015;26 (12):1871-1873

13 Cesmebasi A, Du Plessis M, Iannatuono M, Shah S, Tubbs RS, Loukas M. A review of the anatomy and clinical significance of adrenal veins. Clin Anat 2014;27(8):1253-1263

14 Vonend O, Ockenfels N, Gao X, et al; German Conn's Registry. Adrenal venous sampling: evaluation of the German Conn's registry. Hypertension 2011;57(5):990-995

15 Trerotola SO, Smoger DL, Cohen DL, Fraker DL. The inferior accessory hepatic vein: an anatomic landmark in adrenal vein sampling. J Vasc Interv Radiol 2011;22(9):1306-1311
16 Kohi MP, Agarwal VK, Naeger DM, et al. The inferior emissary vein: a reliable landmark for right adrenal vein sampling. Acta Radiol 2015;56(4):454-457

17 Bajwa R, Bergin D, O'Sullivan GJ. Aiming for the bottom corner: how to score a field goal when landing venous stents in May-Thurner syndrome. J Vasc Interv Radiol 2019;30(10): 1555-1561

18 Lupi A, Battistel M, Barbiero G, Miotto D, Rossi GP, Quaia E. Simultaneous bilateral adrenal vein sampling for primary aldosteronism: useful tips to make it simple and safe. Eur Radiol 2019;29(11):6330-6335

19 Ford KM, Smolinski S, Perez Lozada JC. Anomalous adrenal vein anatomy complicating the evaluation of primary hyperaldosteronism. Radiol Case Rep 2017;13(1):139-141

20 Scholten A, Cisco RM, Vriens MR, Shen WT, Duh Q-Y. Variant adrenal venous anatomy in 546 laparoscopic adrenalectomies. JAMA Surg 2013;148(4):378-383

21 Rossi GP, Barisa M, Allolio B, et al. The Adrenal Vein Sampling International Study (AVIS) for identifying the major subtypes of primary aldosteronism. J Clin Endocrinol Metab 2012;97(5):1606-1614

22 Higashide $\mathrm{T}$, Funabashi $\mathrm{N}$, Tanaka $\mathrm{T}$, et al. Utility of 320 slice mapping CT for adrenal vein sampling in subjects suspected of having primary-aldosteronism compared with digital-subtraction-angiography and selective retrograde CT adrenal venography. Int J Cardiol 2013;168(3):3033-3034

23 Georgiades CS, Hong K, Geschwind J-F, et al. Adjunctive use of C-arm CT may eliminate technical failure in adrenal vein sampling. J Vasc Interv Radiol 2007;18(9):1102-1105

24 Reardon MA, Angle JF, Abi-Jaoudeh N, et al. Intraprocedural cortisol levels in the evaluation of proper catheter placement in adrenal venous sampling. J Vasc Interv Radiol 2011;22(11):1575-1580 